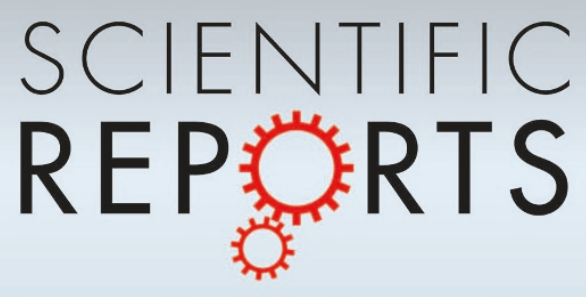

OPEN

SUBJECT AREAS:

MAGNETIC PROPERTIES

AND MATERIALS

CONDENSED-MATTER PHYSICS

ELECTRONIC PROPERTIES AND MATERIALS

Received

16 July 2014

Accepted

9 October 2014

Published

29 October 2014

Correspondence and requests for materials should be addressed to T.T. (t.takayama@fkf. mpg.de)

\section{Spin-orbit coupling induced semi-metallic state in the 1/3 hole-doped hyper-kagome $\mathrm{Na}_{3} \mathrm{Ir}_{3} \mathrm{O}_{8}$}

Tomohiro Takayama ${ }^{1,2}$, Alexander Yaresko' , Akiyo Matsumoto' ${ }^{2}$ Jürgen Nuss' , Kenji Ishiii ${ }^{3}$, Masahiro Yoshida ${ }^{3,4}$, Junichiro Mizuki ${ }^{3,4}$ \& Hidenori Takagi ${ }^{1,2,5}$

${ }^{1}$ Max Planck Institute for Solid State Research, Heisenbergstrasse 1, 70569 Stuttgart, Germany, ${ }^{2}$ Department of Physics and Department of Advanced Materials, University of Tokyo, 7-3-1 Hongo, Bunkyo-ku, Tokyo 113-0033, Japan, ${ }^{3}$ SPring-8, Japan Atomic Energy Agency, Sayo, Hyogo 679-51 48, Japan, ${ }^{4}$ School of Science and Technology, Kwansei Gakuin University, Sanda, Hyogo 669-1337, Japan, ${ }^{5}$ RIKEN Advanced Science Institute, Wako 351-0195, Japan.

The complex iridium oxide $\mathrm{Na}_{3} \mathrm{Ir}_{3} \mathrm{O}_{8}$ with a B-site ordered spinel structure was synthesized in single crystalline form, where the chiral hyper-kagome lattice of Ir ions, as observed in the spin-liquid candidate $\mathrm{Na}_{4} \mathrm{Ir}_{3} \mathrm{O}_{8}$, was identified. The average valence of $\mathrm{Ir}$ is $4.33+$ and, therefore, $\mathrm{Na}_{3} \mathrm{Ir}_{3} \mathrm{O}_{8}$ can be viewed as a doped analogue of the hyper-kagome spin liquid with $\mathrm{Ir}^{4+}$. The transport measurements, combined with the electronic structure calculations, indicate that the ground state of $\mathrm{Na}_{3} \mathrm{Ir}_{3} \mathrm{O}_{8}$ is a low carrier density semi-metal. We argue that the semi-metallic state is produced by a competition of the molecular orbital splitting of $t_{2 g}$ orbitals on $\operatorname{Ir}_{3}$ triangles with strong spin-orbit coupling inherent to heavy Ir ions.

r he realization of quantum spin liquid is a long-sought dream in condensed matter physics, where exotic phenomena such as spinon Fermi surface, fractional excitations or unconventional superconductivity upon doping are anticipated ${ }^{1}$. The most promising arena for spin liquid is in geometrically frustrated lattices based on a triangular motif. Antiferromagnetically interacting spins on such a frustrated lattice cannot simultaneously satisfy all magnetic bonds and the interplay with quantum effect may give rise to a quantum liquid state of spins. To date experimental efforts have yielded several candidates, such as transition metal oxides with a kagome lattice ${ }^{1,2}$ and organic Mott insulators with a triangular lattice ${ }^{1,3}$.

A prime candidate in these recent discoveries is $\mathrm{Na}_{4} \mathrm{Ir}_{3} \mathrm{O}_{8}{ }^{4}$. It is one of the very first candidates for a threedimensional quantum spin liquid, where $\operatorname{Ir}^{4+}$ ions with a localized $S=1 / 2$ moment (or most likely close to $J_{\text {eff }}=1 / 2$ moment, see below) form a corner-sharing network of triangles in three-dimensions, called a "hyper-kagome" lattice. All the Ir sites and the Ir-Ir bonds are equivalent, rendering the hyper-kagome lattice magnetically frustrated. Indeed, $\mathrm{Na}_{4} \mathrm{Ir}_{3} \mathrm{O}_{8}$ exhibits no magnetic ordering down to $2 \mathrm{~K}$ despite the strong antiferromagnetic interaction inferred from the Curie-Weiss temperature $\theta_{\mathrm{W}} \sim-650 \mathrm{~K}$. This discovery triggered intensive experimental and theoretical surveys on this compound, including proposals for the presence of a spinon Fermi surface 5 .

In tandem with the discovery of $\mathrm{Na}_{4} \mathrm{Ir}_{3} \mathrm{O}_{8}$, complex $\mathrm{Ir}^{4+}$ oxides have recently been recognized as a novel playground for physics of strong spin-orbit coupling (SOC). SOC of $\operatorname{Ir}^{4+}$ is as large as $\lambda_{\mathrm{SO}} \sim 0.6 \mathrm{eV}$, reflecting the heavy atomic mass. $\lambda_{\mathrm{SO}}$ is significant even when compared with the other parameters dominating the electronic states, such as the inter-site hopping $t$, the Coulomb repulsion $U$ and the crystal field splitting $\Delta$. The interplay of the large SOC with the other parameters leads to the formation of unprecedented electronic phases. In the layered perovskite $\mathrm{Sr}_{2} \mathrm{IrO}_{4}$, for example, a half filled $J_{\text {eff }}=1 / 2$ state is formed by strong SOC, which is a superposition of three $t_{2 g}$ orbitals $\mid J_{\text {eff }}=1 / 2>=1 / \sqrt{3}(|x y, \pm \sigma> \pm| y z, \mp \sigma>+i \mid x y, \mp \sigma>)$ and gives rise to a spin-orbital Mott insulator ${ }^{6}$. The magnetic coupling of $J_{\text {eff }}=1 / 2$ moments in such spin-orbital Mott insulators can be distinct from those of the spin-dominant moments in $3 d$ oxides, as exemplified by the possible Kitaev spin-liquid proposed for honeycomb iridates ${ }^{7}$. Such unique magnetic couplings in iridates may make the mystery of the possible spinliquid state of $\mathrm{Na}_{4} \mathrm{Ir}_{3} \mathrm{O}_{8}$ even more intriguing ${ }^{8}$.

The insulating state of $\mathrm{Na}_{4} \mathrm{Ir}_{3} \mathrm{O}_{8}$ is marginally stabilized by a modest $U$ with the help of strong SOC. Such weak Mottness, implying the close proximity to a metallic state ${ }^{9}$, has been proposed to play a vital role in realizing the spin-liquid ground state here, as well as in organic triangular spin liquids ${ }^{1}$. It is natural to expect that $\mathrm{Na}_{4} \operatorname{Ir}_{3} \mathrm{O}_{8}$ 
might switch into a metallic state by carrier-doping or by applying pressure. Exotic superconductivity at the critical border to a spin liquid might be anticipated in analogy with the organic systems ${ }^{10}$. The metallic state of hyper-kagome iridate is therefore worthy of exploration.

Despite such intriguing outlooks in $\mathrm{Na}_{4} \mathrm{Ir}_{3} \mathrm{O}_{8}$, not much progress has been achieved in the critical investigation of the spin-liquid like state including the role of spin-orbit coupling, largely due to the lack of single crystals. During the course of attempting to grow single crystals of $\mathrm{Na}_{4} \mathrm{Ir}_{3} \mathrm{O}_{8}$, we obtained single crystals of $\mathrm{Na}_{3} \mathrm{Ir}_{3} \mathrm{O}_{8}$, a B-site ordered spinel. The crystal structure is distinct from that of $\mathrm{Na}_{4} \mathrm{Ir}_{3} \mathrm{O}_{8}$, a spin liquid candidate, but shares the same Ir-O hyper-kagome network. $\mathrm{Na}_{3} \mathrm{Ir}_{3} \mathrm{O}_{8}$ therefore can be viewed as a doped hyper-kagome spin liquid. We report here that $\mathrm{Na}_{3} \mathrm{Ir}_{3} \mathrm{O}_{8}$ has a semi-metallic ground state produced by a subtle competition of strong spin-orbit coupling and molecular orbital splitting of $t_{2 g}$ electrons in an $\mathrm{Ir}_{3}$ triangular unit.

\section{Results}

Crystal Structure Analysis. Single crystals of $\mathrm{Na}_{3} \mathrm{Ir}_{3} \mathrm{O}_{8}$ were grown by a flux method (see Methods). The obtained crystals are stable in air. Detailed refinement of structure using single crystal X-ray diffraction was performed on the crystals. Satisfactory refinement was performed with space groups $P 4_{1} 32$ or $P 4_{3} 32$. The obtained structural parameters are listed in Table 1. The structure shown in Fig. 1a can be viewed as an ordered spinel, an intimately related but distinct structure to that of $\mathrm{Na}_{4} \mathrm{Ir}_{3} \mathrm{O}_{8}$. Rewriting the chemical formula of $1 / 2 \mathrm{Na}_{3} \mathrm{Ir}_{3} \mathrm{O}_{8}$ as $\mathrm{Na}\left(\mathrm{Na}_{1 / 4}, \mathrm{Ir}_{3 / 4}\right)_{2} \mathrm{O}_{4}$, in correspondence with that of spinel $\mathrm{AB}_{2} \mathrm{O}_{4}$, is useful to understand the structure. $\mathrm{Na} 2$ in Table 1 corresponds to the tetrahedral A-site of spinel structure. In $\mathrm{Na}_{4} \mathrm{Ir}_{3} \mathrm{O}_{8}$, this site is empty and, instead, the octahedral A-site is occupied by $\mathrm{Na}_{1.5}$. The occupancies of the octahedral sites were found to be zero in the $\mathrm{Na}_{3} \mathrm{Ir}_{3} \mathrm{O}_{8}$ single crystal within the resolution of structural analysis. Na1 corresponds to $1 / 4$ of the pyrochlore B sub-lattice. The remaining $3 / 4$ of the pyrochlore $B$ sub-lattice sites are occupied by Ir atoms. All the Ir sites are equivalent and form the same hyper-kagome network as in $\mathrm{Na}_{4} \mathrm{Ir}_{3} \mathrm{O}_{8}$. The presence of chirality in the hyper-kagome network gives rise to two possible space groups with different chiralities. The crystals under investigation contained racemic twins (see Supplementary). We did not find any signature of non-stoichiometry such as sodium deficiency in the refinement and therefore the composition of single crystal should be stoichiometric $\mathrm{Na}_{3} \mathrm{Ir}_{3} \mathrm{O}_{8}$.

Transport and Magnetic Properties. The composition of $\mathrm{Na}_{3} \mathrm{Ir}_{3} \mathrm{O}_{8}$ corresponds to Ir valence of $4.33+$, not $4+$. Considering that $\mathrm{Na}_{4} \mathrm{Ir}_{3} \mathrm{O}_{8}$ is an $\mathrm{Ir}^{4+}\left(5 d^{5}\right)$ Mott insulator, $\mathrm{Na}_{3} \mathrm{Ir}_{3} \mathrm{O}_{8}$ may be viewed as $1 / 3$ hole-doped hyper-kagome spin liquid. Indeed, the obtained single crystals were found to show metallic behavior of resistivity as shown in Fig. $2 \mathrm{a}$, in marked contrast to the spin liquid $\mathrm{Na}_{4} \mathrm{Ir}_{3} \mathrm{O}_{8}$. The magnitude of resistivity is relatively large as a metal, $\sim 1 \mathrm{~m} \Omega \mathrm{cm}$ at $5 \mathrm{~K}$. The Hall coefficient of $\mathrm{Na}_{3} \mathrm{Ir}_{3} \mathrm{O}_{8}$ indicates that the poorly metallic behavior originates from a low carrier concentration. The
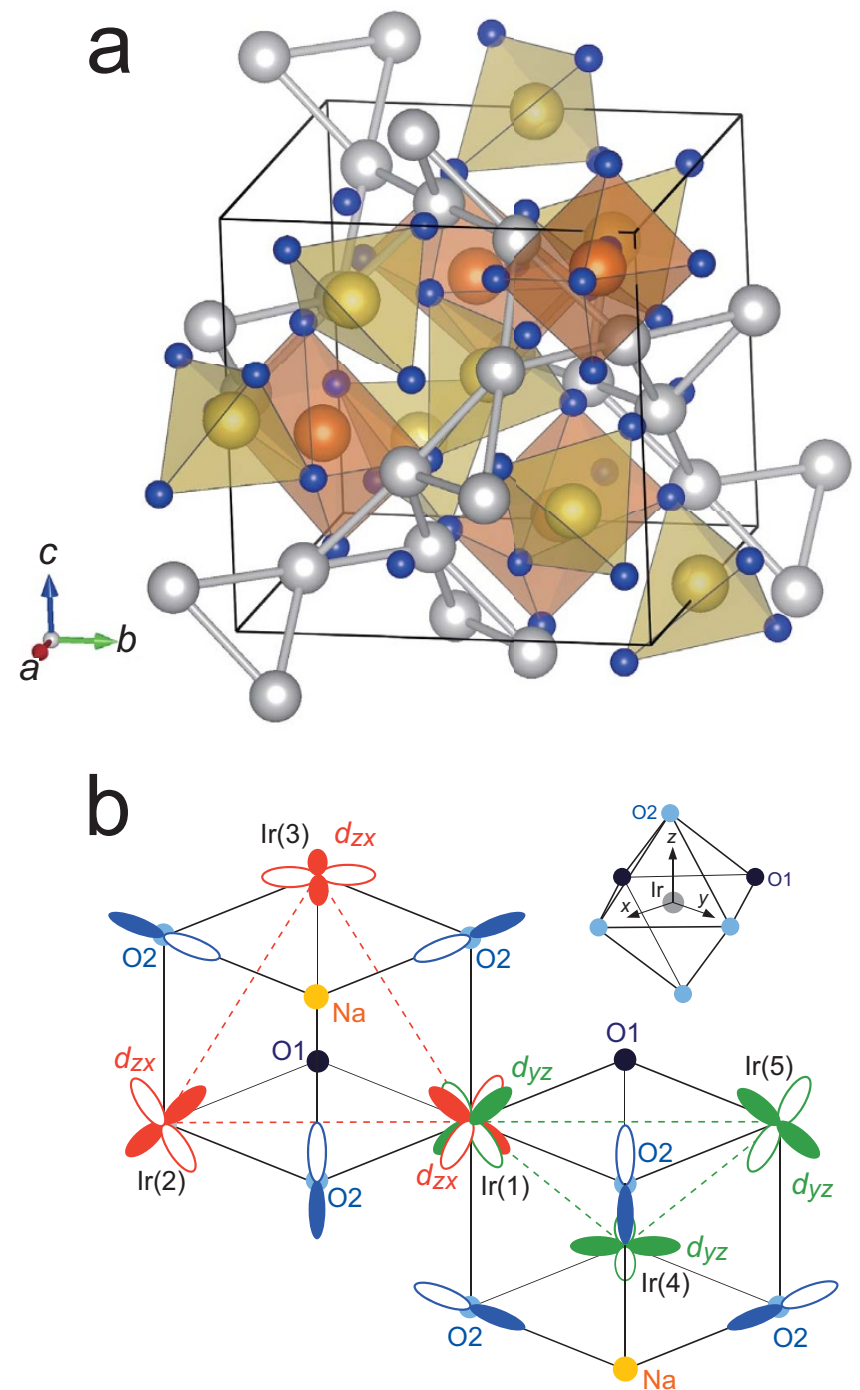

Figure $1 \mid$ Crystal structure of $\mathrm{Na}_{3} \mathrm{Ir}_{3} \mathrm{O}_{8}$. (a) The unit cell of $\mathrm{Na}_{3} \mathrm{Ir}_{3} \mathrm{O}_{8}$ with a space group of $P_{1} 32$. The orange, yellow, silver and blue spheres represent $\mathrm{Na} 1, \mathrm{Na} 2, \mathrm{Ir}$ and $\mathrm{O}$ atoms, respectively ${ }^{19}$. While Na1 atoms are enclosed in distorted $\mathrm{O}_{6}$ octahedra, $\mathrm{Na} 2$ atoms are coordinated tetrahedrally by oxygen atoms. The iridium atoms comprise cornersharing triangles in three-dimensions called hyper-kagome lattice. (b) Schematic illustration of the hyper-kagome lattice showing formation of molecular orbitals on $\mathrm{Ir}_{3}$ triangles seen in the scalar relativistic calculation. $d_{z x}$ on $\operatorname{Ir}(1)$ has hopping paths to $\operatorname{Ir}(2)$ and $\operatorname{Ir}(3)$ via $\mathrm{O} 2$ p-orbitals, forming a localized molecular orbitals. Likewise, $d_{y z}$ on $\operatorname{Ir}(1)$ forms another molecular orbital with the like orbitals on $\operatorname{Ir}(4)$ and $\operatorname{Ir}(5)$. Note that the $t_{2 g}$ orbitals are defined in a local frame (sketched in the inset) which is unique for each Ir site. The hopping via $\mathrm{O} 1$, which is likely much smaller than the one via $\mathrm{O} 2^{17}$, is neglected here.

Table 1 Refined structural parameters of $\mathrm{Na}_{3} \mathrm{Ir}_{3} \mathrm{O}_{8}$. The space group is $P 4_{1} 32$ ( $\mathrm{No} .213$ ) and $Z=4$, and the lattice constant is $a=$ $8.9857(4) \AA$. $g$ and $U_{\text {iso }}$ denote site occupancy and isotropic displacement parameter, respectively. The final $R$ indices are $R=0.0133$ and $w R=0.0287$

\begin{tabular}{|lllcccl|} 
Atom & Site & $g$ & $x$ & $y$ & $z$ & $U_{\text {iso }}\left(\AA^{2}\right)$ \\
\hline rr & $12 d$ & 1 & $0.61264(1)$ & $x+1 / 4$ & $5 / 8$ & $0.00802(4)$ \\
$\mathrm{Nal}$ & $4 b$ & 1 & $7 / 8$ & $7 / 8$ & $7 / 8$ & $0.0122(5)$ \\
$\mathrm{Na2}$ & $8 c$ & 1 & $0.2570(2)$ & $x$ & $x$ & $0.0138(4)$ \\
$\mathrm{O} 1$ & $8 c$ & 1 & $0.1144(2)$ & $x$ & $x$ & $0.0105(6)$ \\
$\mathrm{O} 2$ & $24 e$ & 1 & $0.1364(3)$ & $0.9071(2)$ & $0.9186(2)$ & $0.0111(4)$ \\
\hline
\end{tabular}




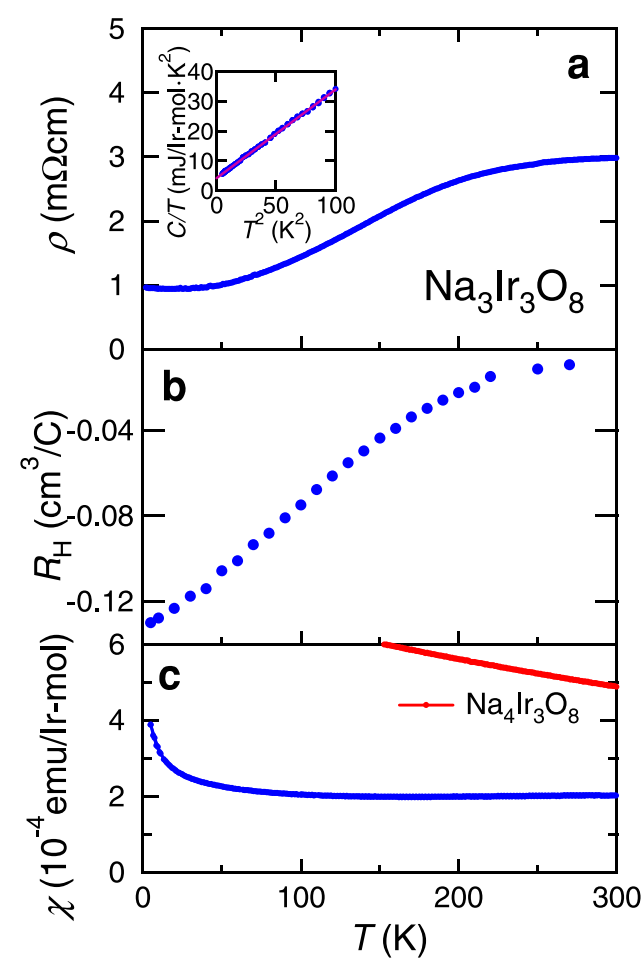

Figure 2 Transport, magnetic and thermodynamic properties of $\mathrm{Na}_{3} \mathrm{Ir}_{3} \mathrm{O}_{8}$. (a) Resistivity, (b) Hall coefficient and (c) Magnetic susceptibility of $\mathrm{Na}_{3} \mathrm{Ir}_{3} \mathrm{O}_{8}$ single crystal. The red dots in (c) represent magnetic susceptibility of $\mathrm{Na}_{4} \mathrm{Ir}_{3} \mathrm{O}_{8}$ polycrystalline sample for comparison. The inset in (a) shows specific heat $C$ divided by temperature $T$ of $\mathrm{Na}_{3} \mathrm{Ir}_{3} \mathrm{O}_{8}$. The solid line is a fit based on the conventional model $C / T=\gamma+\beta T^{2}$.

Hall coefficient shown in Fig. $2 b$ is negative and its magnitude is orders of magnitude larger than those of typical metals, indicating that a very low density of electrons dominates the transport. The carrier number estimated from the Hall constant is of the order of $10^{19} \mathrm{~cm}^{-3}$ at $5 \mathrm{~K}$, which is too small if the picture of a simple $1 / 3$ holedoped Mott insulator with 2/3 electrons is to be assumed. The Hall mobility at $5 \mathrm{~K}$ is as large as $\sim 100 \mathrm{~cm}^{2} / \mathrm{V} \cdot \mathrm{s}$ and, clearly, the disorder is not the dominant factor of the poorly metallic behavior. The Hall coefficient shows a rapid decrease, more than one order of magnitude, with increasing temperature from $5 \mathrm{~K}$ to $300 \mathrm{~K}$, despite the metallic behavior of resistivity. This very likely implies the coexistence of two different types of carriers at least at high temperatures, suggesting that $\mathrm{Na}_{3} \mathrm{Ir}_{3} \mathrm{O}_{8}$ is either a semi-metal or a very narrow gap semiconductor doped with a small number of carriers. The result of band calculation shown below indicates that the former is the case. Unexpectedly, the ground state of a doped hyper-kagome appears to be very close to a band insulator.

The low temperature specific heat of $\mathrm{Na}_{3} \mathrm{Ir}_{3} \mathrm{O}_{8}$ single crystal, shown in the inset of Fig. 2a, yields an electronic specific heat coefficient $\gamma=4.3 \mathrm{~mJ} / \mathrm{Ir}-\mathrm{mol} \cdot \mathrm{K}^{2}$. This value is pronounced especially when the vanishingly small density of carriers is considered and therefore implies the presence of heavy mass carriers. The magnetic susceptibility (Fig. 2c) is almost temperature-independent, distinct from that of magnetic $\mathrm{Na}_{4} \mathrm{Ir}_{3} \mathrm{O}_{8}$. The paramagnetic susceptibility is estimated to be $\chi_{\mathrm{PM}}=2.7 \times 10^{-4} \mathrm{emu} / \mathrm{Ir}$ - $\mathrm{mol}$ by subtracting the contribution of core diamagnetism taken from the values for $\mathrm{Na}^{+}$, $\mathrm{Ir}^{4+}$ and $\mathrm{O}^{2-}$. This value gives the Wilson ratio $R_{\mathrm{W}} \sim 4.6$ combined with $\gamma=4.3 \mathrm{~mJ} / \mathrm{Ir}-\mathrm{mol} \cdot \mathrm{K}^{2}$. The large $R_{\mathrm{W}}$ at a glance would mean a Stoner enhancement and close proximity to ferromagnetism. Considering the small density of carriers, we speculate that the enhanced $\chi_{\mathrm{PM}}$ might be ascribed alternatively to a superposition of Van Vleck-like contribution.
Resonant Inelastic X-ray Scattering. To verify the 1/3 hole-doped state, we performed a resonant inelastic X-ray scattering (RIXS) at $L_{3}$-edge of $\operatorname{Ir}\left(2 p_{3 / 2} \rightarrow 5 d\right)^{11}$ on $\mathrm{Na}_{3} \mathrm{Ir}_{3} \mathrm{O}_{8}$ single crystals and $\mathrm{Na}_{4} \mathrm{Ir}_{3} \mathrm{O}_{8}$ polycrystalline samples. In the obtained RIXS spectra shown in Fig. 3 , three peaks at around 0.2, 1.0 and $4.0 \mathrm{eV}$ are clearly observed. All those peaks show only small dispersions, which very likely originates from the intra-atomic excitations within the $d$-orbitals of Ir. The intra-atomic character of excitations justifies the comparison of spectra for the single crystal $\mathrm{Na}_{3} \mathrm{Ir}_{3} \mathrm{O}_{8}$ and the polycrystalline $\mathrm{Na}_{4} \mathrm{Ir}_{3} \mathrm{O}_{8}$. A clear shift of peaks at $1.0 \mathrm{eV}$ and $4.0 \mathrm{eV}$ to lower energy and a strong suppression of the $0.2 \mathrm{eV}$ peak are clearly seen in $\mathrm{Na}_{4} \mathrm{Ir}_{3} \mathrm{O}_{8}$. The peak at $4.0 \mathrm{eV}$ can be assigned to $t_{2 g} \rightarrow e_{g}$ transitions, whereas the peaks at $1.0 \mathrm{eV}$ and $0.2 \mathrm{eV}$ likely originate from the excitations within the $t_{2 g}$ manifold. The shift of $4.0 \mathrm{eV}$ peak can be ascribed to the increased Ir-O distance $(\sim 3 \%)$ in $\mathrm{Na}_{4} \mathrm{Ir}_{3} \mathrm{O}_{8}$ and hence the reduced cubic splitting $10 \mathrm{Dq}$. The change in the $1.0 \mathrm{eV}$ and $0.2 \mathrm{eV}$ peaks cannot be accounted for simply by the crystal field effect and very likely reflects the difference of band filling between the two compounds. This point will be justified based on the result of band calculation described below, which supports the $1 / 3$ hole-doped state in $\mathrm{Na}_{3} \mathrm{Ir}_{3} \mathrm{O}_{8}$.

Electronic Structure Calculation. $a b$ initio electronic structure calculation using a fully relativistic LMTO code $^{12}$ revealed that $\mathrm{Na}_{3} \mathrm{Ir}_{3} \mathrm{O}_{8}$ is a compensated semi-metal due to the interplay of periodic potential and SOC, which is consistent with the experimental observation described above. Figure 4 depicts the electronic states around the Fermi energy where $t_{2 g}$ orbitals of Ir have a dominant contribution. The $5 d$ electrons are accommodated into the $t_{2 g}$ manifold due to the large $t_{2 g}-e_{g}$ crystal field splitting. The number of $d$-electrons per Ir atom is non-integer, $4.67\left(\mathrm{Ir}^{4.33+}\right)$. In the unit formula with $3 \mathrm{Ir}$ atoms, we have even number of electrons, $14(=3 \times 4.67) t_{2 g}$ electrons. In the scalar-relativistic calculation neglecting SOC, shown in Fig. 4a, a well-defined gap of $0.2 \mathrm{eV}$ can be seen within the $t_{2 g}$ bands. $14 t_{2 g}$ electrons fill up all the bands below the $0.2 \mathrm{eV}$ gap and the system is a band insulator. A similar gap separating $t_{2 g}$ bands and explained by strong $p-d$ hopping was also found for $\mathrm{Na}_{4} \mathrm{Ir}_{3} \mathrm{O}_{8}{ }^{13}$, where, due to the higher $\mathrm{Na}$ content, the unoccupied $t_{2 g}$ bands above the gap in $\mathrm{Na}_{3} \mathrm{Ir}_{3} \mathrm{O}_{8}$ are partially filled.

Strong SOC of Ir, in reality, splits the conduction and the valence bands substantially and a negative band gap is realized. The band structure calculated with SOC is shown in Fig. 4d. Since the crystal structure lacks inversion symmetry, SOC lifts the Kramers degeneracy everywhere except for time-reversal invariant points. In the presence of realistic SOC, a pair of unoccupied $t_{2 g}$ bands, colored in magenta, bends down near the $\mathrm{R}$ point and becomes degenerate

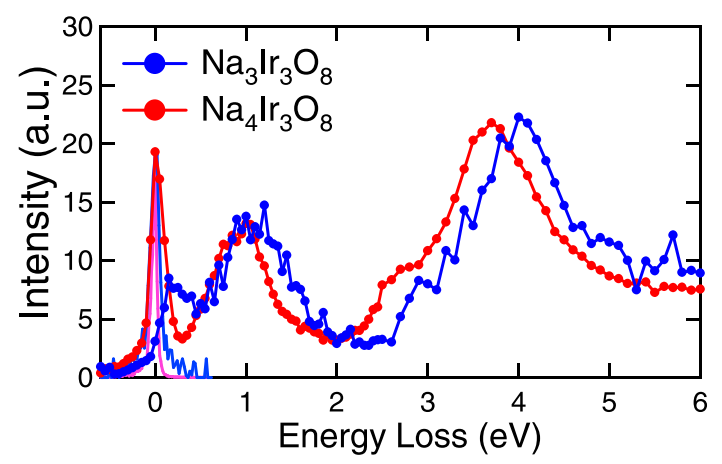

Figure 3 RIXS spectra of $\mathrm{Na}_{3} \mathrm{Ir}_{3} \mathrm{O}_{8}$ single crystal and polycrystalline $\mathrm{Na}_{4} \mathrm{Ir}_{3} \mathrm{O}_{8}$ at $\mathrm{Ir} L_{3}$-edge. The two spectra were normalized by the intensity of the high energy tail above $6 \mathrm{eV}$. The blue and pink solid lines around zero energy show the normalized elastic signals independently measured with $\sigma$-polarized incident X-ray. 

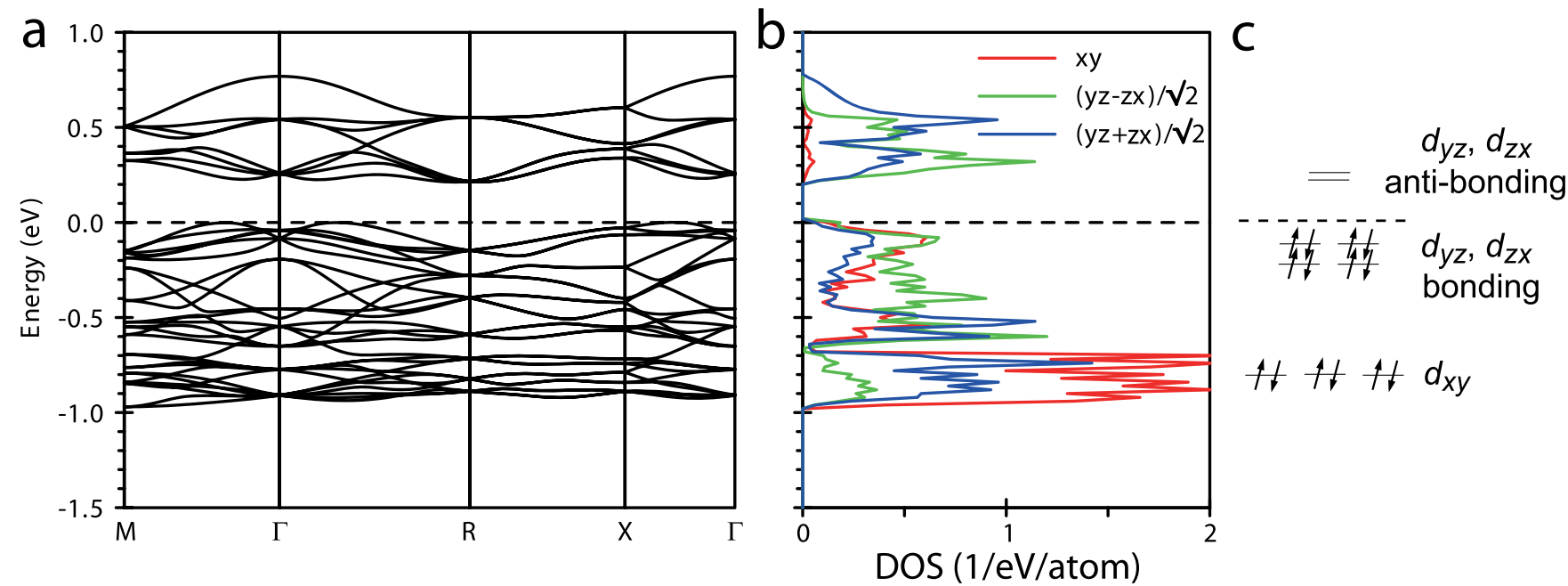

\section{$1 t+1 t d_{x y}$}
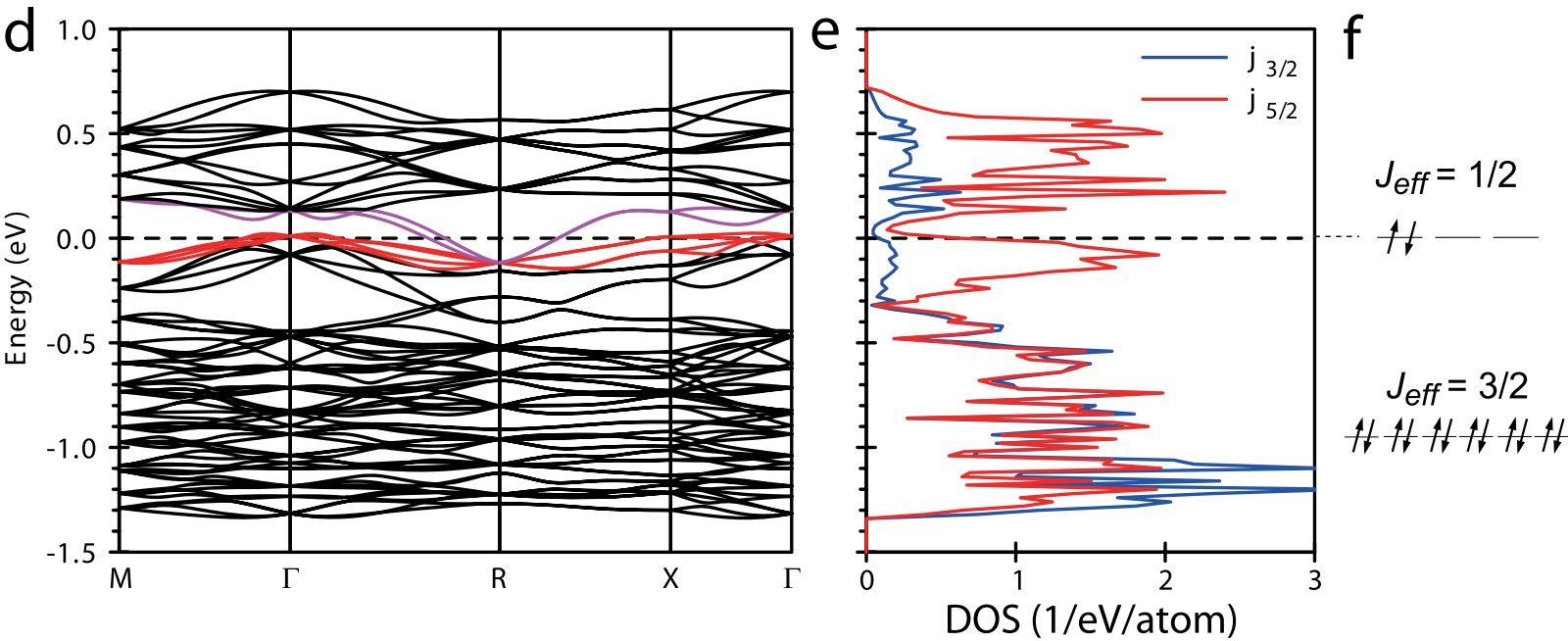

Figure 4 Electronic structure calculation for $\mathrm{Na}_{3} \mathrm{Ir}_{3} \mathrm{O}_{8}$. (a) Scalar-relativistic band structure, and (b) orbital resolved Ir $5 d$ density of states (DOS). The $x y$ orbital represents a $t_{2 g}$ orbital lying in the plane which contains two larger Ir-O1 bonds. (c) Schematic energy level splitting of the $t_{2 g}$ orbitals in an $\mathrm{Ir}_{3}$ triangular molecule. The $d_{x y}$ orbitals have lower energy due to the crystal field. The $d_{y z}$ and $d_{z x}$ orbitals form bonding and anti-bonding molecular orbitals, which produce an energy gap. (d) Relativistic band structure including spin-orbit coupling (SOC). The bands which form hole and electron pockets are colored in red and magenta, respectively. (e) Ir $5 d$ density of states corresponding to (d) resolved with $j=5 / 2$ and $3 / 2$ characters.

(f) Local $d$-level splitting for an $\operatorname{Ir}_{3}$ triangle with SOC. At each Ir atom coordinated octahedrally by $\mathrm{O}$ atoms, SOC splits the $t_{2 g}$ orbitals into lower lying $J_{\text {eff }}$ $=3 / 2$ quartet and upper $J_{\text {eff }}=1 / 2$ doublet. 14 electrons in an $\operatorname{Ir}_{3}$ triangle fills up the $J_{\text {eff }}=3 / 2$ states, while the $J_{\text {eff }}=1 / 2$ states are occupied partially.

with the valence bands at the $\mathrm{R}$ point. This pronounced SOC effect results in the overlap of the pair of conduction band and the valence band colored in red, and creates two electron-like Fermi surfaces around the $\mathrm{R}$ point and four hole-like Fermi surfaces near the $\Gamma$ point. $\mathrm{Na}_{3} \mathrm{Ir}_{3} \mathrm{O}_{8}$ is therefore a robust semi-metal, protected by the degeneracy at the $\mathrm{R}$ point. From the calculation, the masses of carriers are estimated; 1.4, 2.3, 3.7 and $5.6 m_{0}$ for hole bands and $1.8 m_{0}$ for two electron bands. The relatively heavy masses yield a large specific heat coefficient $\gamma_{\text {calc }}=2.9 \mathrm{~mJ} / \mathrm{Ir}-\mathrm{mol} \cdot \mathrm{K}^{2}$, though the carrier density is small. The $\gamma_{\text {calc }}$ is close to and only $35 \%$ smaller than the experimentally observed $\gamma=4.3 \mathrm{~mJ} / \mathrm{Ir}-\mathrm{mol} \cdot \mathrm{K}^{2}$. The more dispersive electron bands with higher mobility should dominate the transport, again consistent with the negative sign of Hall constant experimentally observed. The recent observation of Fano resonances in the phonon spectra of $\mathrm{Na}_{3} \mathrm{Ir}_{3} \mathrm{O}_{8}$ provides further evidence for the semi-metallic state, where the electron-hole excitation continuum interferes with superimposed discrete phonon states ${ }^{14}$.

\section{Discussion}

The effect of SOC leads to the closure of the scalar relativistic gap in $\mathrm{Na}_{3} \mathrm{Ir}_{3} \mathrm{O}_{8}$. An inspection of orbitally resolved densities of Ir $5 d$ states reveals that the gap closure is a consequence of the competition between SOC and the level splitting associated with the formation of molecular orbitals on the $\mathrm{Ir}_{3}$ triangle. We consider $\mathrm{Ir}_{3}$ triangular "molecules" which are the basic structural unit of the hyper-kagome network. The dominant hopping process between Ir $t_{2 g}$ states in the hyper-kagome lattice is the one between the nearest neighbor Ir $d$ orbitals via oxygen $p$ orbitals $\left(t_{p d \pi}\right)$ which is likely much larger than the direct $\mathrm{Ir} d$ - $d$ hopping ${ }^{15,16}$. There are two oxygen sites, $\mathrm{O} 1$ and $\mathrm{O} 2$, in $\mathrm{Na}_{3} \mathrm{Ir}_{3} \mathrm{O}_{8}$, forming the $\mathrm{IrO}_{6}$ octahedra, and the $\mathrm{Ir}-\mathrm{O}$ bond lengths for those two sites are very different (Ir-O1: $2.053 \AA$, Ir-O2: 1.976 or $1.978 \AA$ ). Because of the contrasting Ir-O bond lengths, the hopping via $\mathrm{O} 2$ is expected to be significantly stronger than via $\mathrm{O} 1$ as in the case of $\mathrm{Na}_{4} \mathrm{Ir}_{3} \mathrm{O}_{8}{ }^{17}$. Let us define for each Ir site its own local frame with the $x$ and $y$ axis lying in the plane of the $\mathrm{IrO}_{4}$ square with two more distant O1 ions (see inset of Fig. 1b). Among the three $t_{2 g}$ orbitals of Ir, the $d_{x y}$ orbital should be energetically stabilized by the crystal field associated with the longer Ir-O1 distance. This can be clearly recognized in the orbitally resolved density of states shown in Fig. 4 b. Since $d_{x y}$ bands are fully filled, only $d_{y z}$ and $d_{z x}$ orbitals are responsible for the bands right above and below the $0.2 \mathrm{eV}$ gap.

Both $d_{y z}$ and $d_{z x}$ have the hopping path to $t_{2 g}$ orbitals of the neighboring Ir atoms via $\mathrm{O} 2$ but it is spatially limited. $d_{z x}$ on $\operatorname{Ir}(1)$ 
can hop only to $d_{z x}$ on $\operatorname{Ir}(2)$ or $\operatorname{Ir}(3)$, whereas $d_{y z}$ only to $d_{y z}$ on $\operatorname{Ir}(4)$ or $\operatorname{Ir}(5)$, meaning that for each of the two orbitals the hopping paths via $\mathrm{O} 2$ are restricted to one of the two $\mathrm{Ir}_{3}$ triangles sharing common $\operatorname{Ir}(1)$. In the simplified picture which considers only the hopping via O2 $2 p$ orbitals, therefore, the $d_{z x}$ states form molecular orbitals on the $\operatorname{Ir}(1) \operatorname{Ir}(2) \operatorname{Ir}(3)$ triangle, namely, two degenerate bonding and one anti-bonding molecular orbitals. The $d_{y z}$ on $\operatorname{Ir}(1)$ participates in molecular orbitals on the $\operatorname{Ir}(1) \operatorname{Ir}(4) \operatorname{Ir}(5)$ triangle. Note that $d_{z x}$ and $d_{y z}$ orbitals are nearly orthogonal to each other, which means that those molecular orbitals which belong to different triangles do not interact and are almost localized. Considering the energy levels of the $\mathrm{Ir}_{3}$ molecule with $3 \times 3=9 t_{2 \mathrm{~g}}$ orbitals, $3 d_{x y}$ orbitals have the lowest energy. The other two, $d_{y z}$ and $d_{z x}$, orbitals form 4 bonding and 2 anti-bonding orbitals, as schematically shown in Fig. 4c. With 14 electrons per $\operatorname{Ir}_{3}$ triangle, $3 d_{x y}$ and 4 bonding molecular orbitals are fully filled and the splitting of bonding and anti-bonding molecular orbitals gives rise to an energy gap.

SOC of heavy Ir $5 d$ orbitals is very large $\sim 0.6 \mathrm{eV}$ and may reconstruct the electronic structure. In a cubic environment SOC splits three-fold degenerate $t_{2 g} d$-orbitals into $2: 1$ ratio of lower $J_{\text {eff }}=3 / 2$ (admixture of $j=5 / 2$ and $3 / 2$ characters) and higher $J_{\text {eff }}=1 / 2$ states (purely $j=5 / 2$ character), which accommodate 12 and 6 electrons respectively for the $\mathrm{Ir}_{3}$ triangle, as shown in Fig. $4 \mathrm{f}$. In the strong SOC limit, $J_{\text {eff }}=3 / 2$ states are fully filled up and $J_{\text {eff }}=1 / 2$ states are $1 / 3$ filled. The splitting into $J_{\text {eff }}=3 / 2$ and higher $J_{\text {eff }}=1 / 2$ states can be seen in the relativistic calculation with SOC shown in Figs. 4d, e. The competition between the molecular orbital split and strong SOC should be responsible for suppression of the $0.2 \mathrm{eV}$ gap in the scalar relativistic calculation. This is because SOC mixes all three $t_{2 g}$ states, $d_{z x}$ and $d_{y z}$ forming two distinct molecular orbitals and $d_{x y}$.

Based on the calculated electronic structure, we can reasonably assign the relevant $d$ - $d$ excitations to the peaks observed in the RIXS spectrum in Fig. 3. The peak structure observed around $1.0 \mathrm{eV}$ corresponds to the excitations from the $J_{\text {eff }}=3 / 2$ to the unoccupied $J_{\text {eff }}=$ $1 / 2$ bands. The peak around $0.2 \mathrm{eV}$ originates from the transition between the occupied $J_{\text {eff }}=1 / 2$ bands with remnant molecular bonding character and the unoccupied $J_{\text {eff }}=1 / 2$ bands with remnant antibonding character, likely representing the strong inter-band transition between the pair of flat bands crossing $E_{\mathrm{F}}$. The absence of the $0.2 \mathrm{eV}$ peak in the RIXS spectrum of $\mathrm{Na}_{4} \mathrm{Ir}_{3} \mathrm{O}_{8}$ is fully consistent with the filling of the unoccupied and flat $J_{\text {eff }}=1 / 2$ bands associated with the removal of $1 / 3$ hole and opening of Mott gap in $\mathrm{Na}_{4} \mathrm{Ir}_{3} \mathrm{O}_{8}$.

In summary, we successfully synthesized single crystals of hyperkagome iridate $\mathrm{Na}_{3} \mathrm{Ir}_{3} \mathrm{O}_{8}$. Unlike the spin-orbital Mott insulator $\mathrm{Na}_{4} \mathrm{Ir}_{3} \mathrm{O}_{8}, \mathrm{Na}_{3} \mathrm{Ir}_{3} \mathrm{O}_{8}$ was found to be a semi-metal. The electronic structure calculation indicates that $\mathrm{Na}_{3} \mathrm{Ir}_{3} \mathrm{O}_{8}$ should be a band insulator with absence of SOC. The semi-metallic state is shown to arise from a competition between the splitting of molecular orbitals on $\mathrm{Ir}_{3}$ triangles and strong SOC. The semi-metallic $\mathrm{Na}_{3} \mathrm{Ir}_{3} \mathrm{O}_{8}$ might be an intriguing platform to test the possible non-trivial topological effects, associated with the frustrated and chiral geometry of the lattice and the negative gap produced by SOC. As an example, the breaking of inversion symmetry due to the chirality, together with strong SOC, produces a sizable Rashba splitting with a Dirac dispersion at the $\Gamma$ point in the hole band, which harbors non-trivial Berry phase in electron transport ${ }^{18}$. We therefore believe that such material inheriting strong SOC and a unique lattice topology provides a tool to substantialize novel states of matter.

\section{Method}

Crystal growth and characterizations. Single crystals of $\mathrm{Na}_{3} \mathrm{Ir}_{3} \mathrm{O}_{8}$ were grown by a flux-growth technique. The mixture of $\mathrm{Na}_{2} \mathrm{CO}_{3}, \mathrm{IrO}_{2}$ and $\mathrm{NaCl}$ with a ratio of $5: 1: 20$ was loaded in an alumina crucible, and heated up to $1075^{\circ} \mathrm{C}$ in an oxygen atmosphere. It was then slowly cooled to $1000^{\circ} \mathrm{C}$ with a cooling rate of $2^{\circ} \mathrm{C} /$ hour, and subsequently furnace-cooled down to room temperature. Black and block-shaped single crystals were found in the solidified melt. The polycrystalline samples of $\mathrm{Na}_{4} \mathrm{Ir}_{3} \mathrm{O}_{8}$ were synthesized by a conventional solid state reaction as a reference material. The crystal structure of single crystals was analyzed by X-ray diffraction using a three circle diffractometer (Bruker AXS) equipped with SMART APEX II CCD, and Mo Ka radiation (see Supplementary). Transport, magnetic and thermodynamic properties were measured with Quantum Design PPMS and MPMS.

In the obtained crystals, we in fact found a very small amount of insulating crystals besides the majority of metallic ones. The X-ray diffraction indicated that the insulating crystals also had the $\mathrm{Na}_{3} \mathrm{Ir}_{3} \mathrm{O}_{8}$ stoichiometry, and the spectroscopic measurements showed no difference between the metallic and insulating crystals. Judging from the poor quality of the insulating crystals, we suspect that the insulating crystals include domains of the $\mathrm{Na}_{4} \mathrm{Ir}_{3} \mathrm{O}_{8}$ phase and there might be a temperature window where $\mathrm{Na}_{4} \mathrm{Ir}_{3} \mathrm{O}_{8}$ phase is stabilized during the crystal growth. However, the growth of $\mathrm{Na}_{4} \mathrm{Ir}_{3} \mathrm{O}_{8}$ single crystal is a future perspective and out of the scope of this article.

Resonant inelastic X-ray scattering (RIXS). Resonant inelastic X-ray scattering (RIXS) measurement was performed at BL11XU of SPring-8. Incident X-rays were monochromatized by a double-crystal $\mathrm{Si}(111)$ monochromator and a secondary $\mathrm{Si}(844)$ channel-cut monochromator. Horizontally scattered X-rays were analyzed by a diced and spherically-bent $\mathrm{Si}(844)$ crystal. The total energy resolution was $70 \mathrm{meV}$. The energy of the incident X-ray was tuned at $11.214 \mathrm{keV}$, which corresponds to the $L_{3}$-edge of $\operatorname{Ir}\left(2 p_{3 / 2} \rightarrow t_{2 g}\right)$.

The samples used were single crystal $\mathrm{Na}_{3} \mathrm{Ir}_{3} \mathrm{O}_{8}$ and polycrystalline $\mathrm{Na}_{4} \mathrm{Ir}_{3} \mathrm{O}_{8}$. The spectra were collected at $20 \mathrm{~K}$ in $\mathrm{Na}_{3} \mathrm{Ir}_{3} \mathrm{O}_{8}$, whereas at $300 \mathrm{~K}$ in $\mathrm{Na}_{4} \mathrm{Ir}_{3} \mathrm{O}_{8}$. The difference in the measurement temperature should not change the overall spectra. The contribution of elastic scattering was evaluated by independently measuring the elastic signal with a $\sigma$-polarized configuration. It was almost negligible in $\mathrm{Na}_{3} \mathrm{Ir}_{3} \mathrm{O}_{8}$, whereas the strong quasi-elastic signal was seen around zero energy in the $\mathrm{Na}_{4} \mathrm{Ir}_{3} \mathrm{O}_{8}$ polycrystalline sample. This is possibly attributed to the elastic signal coming from concomitant Bragg reflections and/or to the phonon excitations inherent to the measurement at room temperature.

1. Balents, L. Spin liquids in frustrated magnets. Nature 464, 199-208 (2010)

2. Mendels, P. \& Bert, F. Quantum kagome antiferromagnet $\mathrm{ZnCu}_{3}(\mathrm{OH})_{6} \mathrm{Cl}_{2}$. Journal of the Physical Society of Japan 79, 011001 (2010).

3. Shimizu, Y., Miyagawa, K., Kanoda, K., Maesato, M. \& Saito, G. Spin liquid state in an organic Mott insulator with a triangular lattice. Phys. Rev. Lett. 91, 107001 (2003).

4. Okamoto, Y., Nohara, M., Aruga-Katori, H. \& Takagi, H. Spin-liquid state in the $S$ $=1 / 2$ hyperkagome antiferromagnet $\mathrm{Na}_{4} \mathrm{Ir}_{3} \mathrm{O}_{8}$. Phys. Rev. Lett. 99, 137207 (2007).

5. Lawler, M. J., Paramekanti, A., Kim, Y. B. \& Balents, L. Gapless spin liquids on the three-dimensional hyperkagome lattice of $\mathrm{Na}_{4} \mathrm{Ir}_{3} \mathrm{O}_{8}$. Phys. Rev. Lett. 101, 197202 (2008).

6. Kim, B. J. et al. Phase-sensitive observation of a spin-orbital Mott state in $\mathrm{Sr}_{2} \mathrm{IrO}_{4}$ Science 323, 1329-1332 (2009).

7. Jackeli, G. \& Khaliullin, G. Mott insulators in the strong spin-orbit coupling limit: from Heisenberg to a quantum compass and Kitaev models. Phys. Rev. Lett. 102, 017205 (2009).

8. Chen, G. \& Balents, L. Spin-orbit effects in $\mathrm{Na}_{4} \mathrm{Ir}_{3} \mathrm{O}_{8}$ : A hyper-kagome lattice antiferromagnet. Phys. Rev. B 78, 094403 (2008).

9. Podolsky, D., Paramekanti, A., Kim, Y. B. \& Senthil, T. Mott transition between a spin-liquid insulator and a metal in three dimensions. Phys. Rev. Lett. 102, 186401 (2009).

10. Kurosaki, Y., Shimizu, Y., Miyagawa, K., Kanoda, K. \& Saito, G. Mott transition from a spin liquid to a Fermi liquid in the spin-frustrated organic conductor $\kappa$ $(\mathrm{ET})_{2} \mathrm{Cu}_{2}(\mathrm{CN})_{3}$. Phys. Rev. Lett. 95, 177001 (2005).

11. Ishii, K. et al. Momentum-resolved electronic excitations in the Mott insulator $\mathrm{Sr}_{2} \mathrm{IrO}_{4}$ studied by resonant inelastic X-ray scattering. Phys. Rev. B 83, 115121 (2011).

12. Antonov, V., Harmon, B. \& Yaresko, A. Electronic Structure and Magneto-Optical Properties of Solids (Kluwer Academic Publishers, Dordrecht, Boston, London, 2004)

13. Norman, M. R. \& Micklitz, T. Electronic structure of hyper-kagome $\mathrm{Na}_{4} \mathrm{Ir}_{3} \mathrm{O}_{8}$. Phys. Rev. B 81, 024428 (2010).

14. Pröpper, D. et al. Fano resonance in the infrared spectra of phonons in hyperkagome $\mathrm{Na}_{3} \mathrm{Ir}_{3} \mathrm{O}_{8}$. Phys. Rev. Lett. 112, 087401 (2014).

15. Shitade, A. et al. Quantum spin Hall effect in a transition metal oxide $\mathrm{Na}_{2} \mathrm{IrO}_{3}$ Phys. Rev. Lett. 102, 256403 (2009).

16. Mazin, I. I., Jeschke, H. O., Foyetsove, K., Valenti, R. \& Khomskii, D. I. $\mathrm{Na}_{2} \mathrm{IrO}_{3}$ as a molecular orbital crystal. Phys. Rev. Lett. 109, 197201 (2012).

17. Micklitz, T. \& Norman, M. R. Spin Hamiltonian of hyper-kagome $\mathrm{Na}_{4} \mathrm{Ir}_{3} \mathrm{O}_{8}$. Phys. Rev. B 81, 174417 (2010).

18. Murakawa, H. et al. Detection of Berry's phase in a bulk Rashba semiconductor. Science 342, 1490-1493 (2013).

19. Momma, K. \& Izumi, F. Vesta 3 for three-dimensional visualization of crystal, volumetric and morphology data. J. Appl. Cryst. 44, 1272-1276 (2011).

\section{Acknowledgments}

We thank A. W. Rost and A. Kato for technical supports in the RIXS measurement and D. Casa for fabrication of $\mathrm{Si}(844)$ analyzer for RIXS. We are grateful to R. Perry, G. Khaliullin, G. Jackeli, A. Bangura, D. Pröpper, A. V. Boris and B. Keimer for fruitful discussion. This work was partly supported by Grant-in-Aid for Scientific Research (S) (Grand No. 
24224010) and Grant-in-Aid for Young Scientists (B) (Grant No. 23740246). The synchrotron radiation experiments were performed at the BL11XU of SPring- 8 with the approval of the Japan Synchrotron Radiation Research Institute (JASRI) (Proposal No. 2012B3502 and 2013A3502).

\section{Author contributions}

T.T. and H.T. conceived and designed the project. T.T. and A.M. performed synthesis and magnetic and transport measurements. J.N. analyzed the crystal structure. K.I., M.Y., T.T., A.M. and J.M. measured and analyzed the RIXS data. A.Y. conducted electronic structure calculation. T.T., A.Y. and H.T. wrote the manuscript and all authors discussed and reviewed the paper.

\section{Additional information}

Supplementary information accompanies this paper at http://www.nature.com/ scientificreports

Competing financial interests: The authors declare no competing financial interests.

How to cite this article: Takayama, T. et al. Spin-orbit coupling induced semi-metallic state in the 1/3 hole-doped hyper-kagome $\mathrm{Na}_{3} \mathrm{Ir}_{3} \mathrm{O}_{8}$. Sci. Rep. 4, 6818; DOI:10.1038/srep06818 (2014).

(i) This work is licensed under a Creative Commons Attribution 4.0 International License. The images or other third party material in this article are included in the article's Creative Commons license, unless indicated otherwise in the credit line; if the material is not included under the Creative Commons license, users will need to obtain permission from the license holder in order to reproduce the material. To view a copy of this license, visit http://creativecommons.org/licenses/by/4.0/ 